\title{
Solving structures by native SAD at room temperature
}

\author{
Andreas Förster ${ }^{a}$, Pascal Hofer $^{b}$ and Clemens Schulze-Briese ${ }^{c}$ \\ ${ }^{a}$ DECTRIS Ltd., Täfernweg 1, 5405 Baden-Dättwil, Switzerland, andreas.foerster@dectris.com \\ ${ }^{b}$ DECTRIS USA Inc., 325 Chestnut Street, Suite 800, Philadelphia, PA, USA, pascal.hofer@dectris.com \\ 'DECTRIS Ltd., Täfernweg 1, 5405 Baden-Dättwil, Switzerland, clemens.schulze-briese@dectris.com
}

Most proteins require flexibility to perform their cellular functions at physiological temperatures (between approximately 270 and $370 \mathrm{~K}$, depending on the organism's habitat). In contrast, structural studies based on electron cryo-microscopy and X-ray crystallography are predominantly performed at temperatures around $100 \mathrm{~K}$. While the overall shape of a protein won't change upon cooling, small but physiologically important details might.

Structural information from samples held at physiological temperatures is required to address this problem. Solution methods like nuclear magnetic resonance spectroscopy and small-angle X-ray scattering can provide some of that information but have other limitations (low molecular weight and resolution, respectively). For X-ray crystallography at physiological temperatures, radiation damage is the biggest impediment. Mitigating this by serial crystallography at free electron lasers or synchrotrons requires enormous resources.

Here we show how accurate data can be collected at room temperature in the laboratory. We collected data from a number of different single crystals at a quality high enough for successful experimental phasing based on the intrinsic anomalous signal (native SAD). Radiation doses were kept to a few dozen $k G y$ to minimize structural damage that would make the observed anomalous differences unreliable.

We show that laboratory X-ray sources with their limited flux in combination with noise-free photon counting detectors are excellent environments for solving protein structures at room temperature. The resulting structures show proteins as close to their physiological state as crystallography can provide. 